\section{Commentary: A new Fontan commandment?}

\author{
Bahaaldin Alsoufi, MD
}

Since its inception in 1971, the Fontan procedure has become the mainstay of surgical palliation of singleventricle anomalies. ${ }^{1}$ Over the years, several surgical modifications have been adopted with the aim to address some of the shortcomings associated with the original descriptions of the procedure. ${ }^{2}$ The Fontan "10 commandments" were first defined by Choussat and colleagues ${ }^{3}$ in 1978 as the basic criteria that the patient should meet to predict successful Fontan palliation. These commandments served for many years as guidelines for physicians to select patients who will be advanced toward the last stage of palliation: the Fontan procedure. The original 10 commandments $^{3}$ were the following: age more than 4 years, normal heart rhythm, normal right atrial volume, normal ventricular function, normal systemic venous return, no atrioventricular valve leak, adequate pulmonary artery size, no distortion of pulmonary arteries from prior surgery, low pulmonary artery pressure (PAP) less than $15 \mathrm{~mm} \mathrm{Hg}$, and low pulmonary vascular resistance index (PVRI) less than 4 Wood units $/ \mathrm{m}^{2}$. Nonetheless, surgical advances, along with better understanding of Fontan physiology and improved perioperative and outpatient management, have allowed physicians to break many of these commandments with only a few currently considered essential for the success of Fontan procedure. In general, ventricular function, PAP, and PVRI constitute the most currently relevant remaining commandments for patient selection.

\footnotetext{
From the Department of Cardiovascular and Thoracic Surgery, University of Louisville School of Medicine, Norton Children's Hospital, Louisville, Ky.

Disclosures: The author reported no conflicts of interest.

The Journal policy requires editors and reviewers to disclose conflicts of interest and to decline handling or reviewing manuscripts for which they may have a conflict of interest. The editors and reviewers of this article have no conflicts of interest.

Received for publication Jan 11, 2022; revisions received Jan 11, 2022; accepted for publication Jan 11, 2022; available ahead of print Jan 14, 2022.

Address for reprints: Bahaaldin Alsoufi, MD, Department of Cardiovascular and Thoracic Surgery, University of Louisville School of Medicine, Norton Children's Hospital, 201 Abraham Flexner Way, Suite 1200, Louisville, KY 40202 (E-mail: balsoufi@hotmail.com).

J Thorac Cardiovasc Surg 2022;164:781-2

$0022-5223 / \$ 36.00$

Copyright (c) 2022 by The American Association for Thoracic Surgery

https://doi.org/10.1016/j.jtcvs.2022.01.013
}

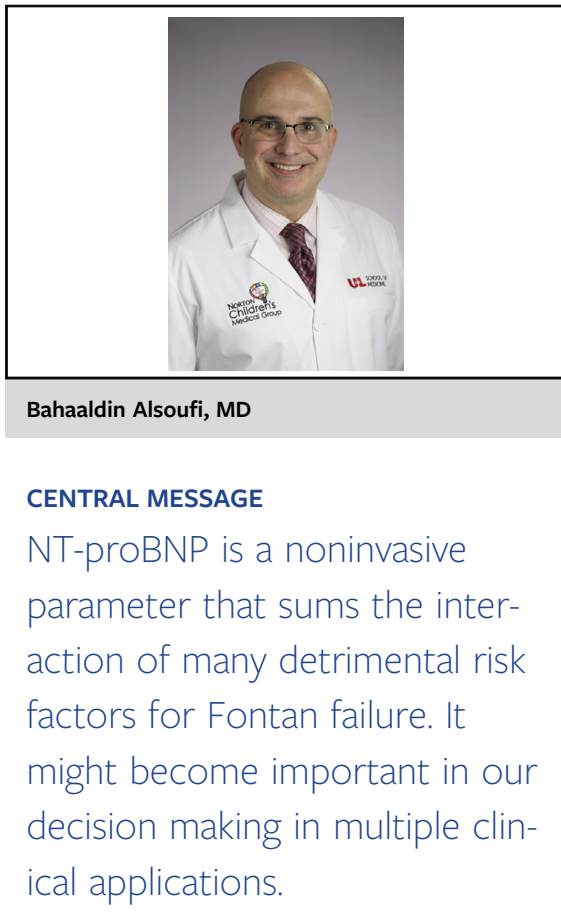

In the current issue of the Journal, Lin and colleagues ${ }^{4}$ from Taiwan present an interesting study in which they examined the role of preoperative $\mathrm{N}$-terminal pro-brain natriuretic peptide (NT-proBNP) level in predicting outcomes after the Fontan procedure. They found that abnormal ventricular function, elevated PAP, high PVRI, and high $\log _{10}$ NT-proBNP level were associated with Fon$\tan$ failure (defined as death, Fontan takedown or heart transplantation) on univariate analysis. Additionally, they found that atrioventricular valve regurgitation moderate or more, elevated PAP, high PVRI, and high $\log _{10}$ NT-proBNP level were associated with rehospitalization due to heart failure on univariate analysis. However, on multivariable Cox regression analysis, $\log _{10}$ NT-proBNP was the only constant significant predictor of adverse outcomes: Fontan failure, rehospitalization for heart failure, and tachyarrhythmias. According to the receiver operating characteristic curve, the best cut point of NT-proBNP value for predicting Fontan failure was $280 \mathrm{pg} / \mathrm{mL}$. According to the receiver operating characteristic curves and hazard ratios, the authors created a scoring system that included 3 preoperative factors (PAP, PVRI, and NT-proBNP) and found that this scoring system was more accurate in predicting Fontan failure than a model without NT-proBNP. In that score, the patient is assigned a score of 0 or 1 for each of the factors if 
the value is less than or greater than $15 \mathrm{~mm} \mathrm{Hg}$ for PAP, 1.6 $\mathrm{WU} \cdot \mathrm{m}^{2}$ for PVRI, and $280 \mathrm{pg} / \mathrm{mL}$ for NT-proBNP. For example, the highest risk patient would have a score of 3 , and that was associated with an annual incidence of Fontan failure of $21 \%$. Another application of NT-proBNP that the authors suggested was guiding the need for Fontan fenestration because a value greater than $280 \mathrm{pg} / \mathrm{mL}$ was predictive of benefit from fenestration, whereas fenestration was not beneficial if the value was less than $280 \mathrm{pg} / \mathrm{mL}$.

The brain natriuretic peptide is a peptide that is released from the heart in response to tissue hypoxia and stretching of the ventricular cardiomyocyte due to volume loading. It is synthesized as a prohormone (proBNP) and on secretion is cleaved into the biologically active brain natriuretic peptide and biologically inert NT-proBNP. Therefore, measurement of NT-proBNP is an indicative of tissue hypoxia and cardiomyocyte stretch. ${ }^{5}$ Although the association of NT-proBNP with various forms of heart failure in adults and children is well established, the authors from Taiwan provide a helpful insight to a novel use of this noninvasive test to predict Fontan failure. NT-proBNP can be obtained noninvasively, and the authors found that NT-proBNP was a superior prognostic parameter than the traditionally used parameters such as PAP, PVRI, or dominant ventricular ejection fraction. It seems that NT-proBNP might be the parameter that computes the interaction of various detrimental hemodynamic and anatomic factors of valvular, myocardial, and pulmonary origin that could contribute in variable degrees to Fontan failure. The authors suggest that NT-proBNP can help guiding surgical management, such as the need for fenestration. Additional potential applications might include guiding the need and assessing the success of atrioventricular or semilunar repair in patients with single-ventricle physiology, adjusting the medical management of suboptimal patients who have undergone the Fontan, and directing the need for Fontan reintervention or listing for transplantation. More research is necessary to understand how to incorporate this parameter in our clinical practice and decision making. Meanwhile, it seems likely that NT-proBNP might soon become the new Fontan commandment in the management of patients with single-ventricle physiology.

\section{References}

1. Fontan F, Baudet E. Surgical repair of tricuspid atresia. Thorax. 1971;26:240-8.

2. de Leval MR, Deanfield JE. Four decades of Fontan palliation. Nat Rev Cardiol. 2010;7:520-7.

3. Choussat A, Fontan I, Besse P, Vallot F, Chauve A, Bricaud H. Selection criteria for Fontan's procedure. In: Anderson RH, Shinebourne EA, eds. Pediatric Cardiology. Edinburgh: Churchill Livingstone; 1978:559-66.

4. Lin H-C, Huang S-C, Wu M-H, Wang J-K, Lin M-T, Chen C-A, et al. Preoperative N-terminal pro-brain natriuretic peptide is associated with Fontan outcomes. $J$ Thorac Cardiovasc Surg. 2022;164:770-80.e3.

5. Hall C. NT-ProBNP: the mechanism behind the marker. J Card Fail. 2005;11: S81-3. 